\title{
The Network Educational Resources Construction on Meteorology Based on Data Mining
}

\author{
Rui Chen, Yang Zhao \\ The China Meteorological Administration Training Centre, Beijing, China \\ Email: crocodilechen sun@163.com
}

Received December 2014

\begin{abstract}
With the vigorous development of the modern network education technology, the construction of network resource developed towards digital, intelligent and personalized, in order to satisfy the distance learners of different learning needs. By analyzing the problems currently learners in the use of the repository for learning generated by the proposed use of data mining techniques to further planning and construction of the repository, and on how to improve the organizational structure of the repository, and improve the utilization of the repository, personalized to meet the current in-depth research and analysis of learning problems.
\end{abstract}

Keywords

Meteorological Education Resource Library, Data Mining, Network Education, Individualized Learning

\section{Introduction}

With the advent of informational times, the weather industry due to the development needs of modern business, open learning model has become a trend, the use of Network Education resource library of information provided at any time, any place, any person, any way of learning while according to different professions, different positions, different regions and even employees have different learning interests and learning objectives for education resources has different requirements, user needs is the highest reference value, high-quality personalized information, in order to better adapt to the user, the repository must be based on the user's state builders, study the user's status, habits and interests, providing high-quality education and training resources, to provide users with a more personalized education integrated services.

\section{The Situation of Meteorological Network Education Resource Library}

Currently, the weather Internet distance education teaching resources include the following:

1) Material resource: Multimedia Library, courseware library, learning parts library, case library, electronic literature, exam etc.; 
2) Online courses: courseware library, school library parts, electronic lesson plans, interactive laboratories, virtual reality laboratory;

3) Teaching Resources Management System: courseware system, teaching observation systems, intelligent tutoring systems, video-on-demand systems, self-examination system, electronic preparation system, electronic library, courseware authoring tools, system test paper and analysis system;

4) General teaching system support platform: electronic preparation system, information distribution system, video conferencing systems, education statistics systems.

\section{The Methods of Education Training Data Mining}

The task of data mining is to extract from the mass of data which is implicit in the process of useful information and knowledge to help users of data for statistical analysis, as well as the sum of reasoning, and thus the use of existing data to predict the future to help trainees learn analysis. Specific tasks include data mining concept description, classification, clustering, correlation, regression and prediction.

Data mining applications in the repository construction, the basic procedure is divided into the following four steps [1] [2]:

1) Extracting web information collected learner characteristics. Information can be collected roughly: learner's identity information, including the learner's name, sex, nationality, age, education level, and address. Using Web logging learner behavior information, such as residence time, number of visits, downloads, and other information hits knowledge.

2) Reprocessing of data, that the source data processing and tissue remodeling, building a data warehouse related topics, provide the basic platform for the next phase of data mining process. Good preparation which includes: data cleaning, data integration, data conversion and data reduction and establish a database mining, and data analysis, data mining can adjust the tasks can be divided into feature extraction, correlation analysis, property classification, clustering analysis and time series forecasting several categories.

3) Analysis and evaluation of test data obtained by mining the knowledge model.

4) Knowledge representation, make full use of the appropriate form to use data mining tools from the web data mining out of the knowledge model shown to improve the construction of the repository.

\section{Data Mining Technology in the Construction of a Network Library}

Using data mining technology to build Network Education Library can effectively address the following three aspects [3]:

1) First let more learners to understand the Library, especially potential users of the excavation. Mining potential users through the analysis of user access to information, the use of classification techniques, to identify the user with a description of some of the public has been classified and old customers to determine the user type; you can dynamically show the user some specific, targeted of the page, to attract users. Learners will be able to know the browsing behavior, to understand the interests and needs of learners, such as the use of data mining, sequential pattern discovery techniques, learners can be found on the page in the form of site navigation behavior, dynamic adjustment of structural sites for the learner, so that students have access to the files associated with the connection between the direct comparison, so that the user can easily access to the page to access, increase in access convenience, better left to the user impression, increase the chance of the next visit. For example, by analyzing Web log mining results [4].

2) Improving resource organization structure to improve resource structure, personalized service is the highest goal of Library construction. By analyzing Web log data, learners learn the history and results of the evaluation and so on, dig out the user's usage patterns to provide personalized services to users; enable learners need to learn the interface more easily accessible, so that learners concern,, link access frequency, access time, the access route using the statistical methods of statistical pages, into the site where the learner to understand is that each home have been, or by direct access to the search engine web page of interest, in which page of the site after the jump.

3) Increasing download speeds by mining association rules in the database, you can achieve the previously stored functions and solve the problem of slow downloads. If each transaction learner personalized access the original database, there are many links to browse the Web while browsing the web are A and B. If a user visits a web browsing a, then B browsing the user is likely to be of value, so you can browse the web together in the 
recommended list B [5].

The mining analysis results into a personalized database, when the learner enters the system, the system can be provided to meet the learning needs of its pages according to the database next time. In addition, due to the learning needs of learners are constantly changing, so the need to regularly collect raw data, combined with personalized learner historical information in the database [6].

\section{Application Examples}

We have statistics of meteorological distance education network in China visited pages; the average residence time longer page information is as Table 1 below.

We can see from Table 1, in meteorological education and training site, respondents' page is mainly resource page, the page residence time in about 4 minutes. From the above data we can see that the operation time of the user most stay in play streaming media courseware, but also can be seen, the streaming media courseware user experience time is about 4 minutes. if the courseware playing time is too long, it will affect the user experience and feelings. From the directory can be seen, most of the resources are concentrated in the path of http://www.cmatc.cn/www/res/page, therefore we could infer that most users are concentrated to stay in the choice of resources, and then mapped to different pages, and eventually to view resources information services. After analysis we will cluster them into a group, and fully understand the multimedia content which these learners interested in, timely adjust the page and page content. In the content present to these learners', increase the content and links to multimedia knowledge, meet the needs of learners.

In the process of implementation of the page, according to the web log, we need to understand the flow distribution of main page Jump, which has important significance to the redirection of resources. From Figure 1 we can clearly see that, turning each page is different, from the jump of the page, for strong steering courseware resources and teaching resources.

The access information of a fixed professional resources page is as Figure 2.

It is shown in Figure 2, the university academic staff have more requirements for the resource, the number of activities and visits are also much more than others, which is related to the number of employees and professionals of the meteorological system. The majority of training staff are undergraduates, at the same time for their resource demands more, pointing to customize more resources to them, for customized service.

Table 1. Statistical ranking table of the visit on the ten websites of one day.

\begin{tabular}{clc}
\hline No. & \multicolumn{1}{c}{ Visited pages } & Residence time \\
\hline 1 & $\underline{\text { http://www.cmatc.com.cn/www/res/xjz/index.shtml }}$ & $5 \mathrm{~min} 20 \mathrm{~s}$ \\
2 & $\underline{\text { http://www.cmatc.cn/www/res/xykw/index.shtml }}$ & $3 \mathrm{~min} 10 \mathrm{~s}$ \\
3 & $\underline{\text { http://www.cmatc.cn/www/res/kjgp/index.shtml }}$ & $2 \mathrm{~min} 10 \mathrm{~s}$ \\
4 & $\underline{\text { http://stream1.cma.gov.cn/cmatcvod/12/nyqxgc/ }}$ & $2 \mathrm{~min} 5 \mathrm{~s}$ \\
5 & $\underline{\text { http://www.cmatc.cn/www/res/jzhmsh/index.shtml }}$ & $1 \mathrm{~min} 40 \mathrm{~s}$ \\
6 & $\underline{\text { http://www.cmatc.cn/www/res/yuanchengpeixun/index.shtml }}$ & $1 \mathrm{~min}$ \\
\hline
\end{tabular}

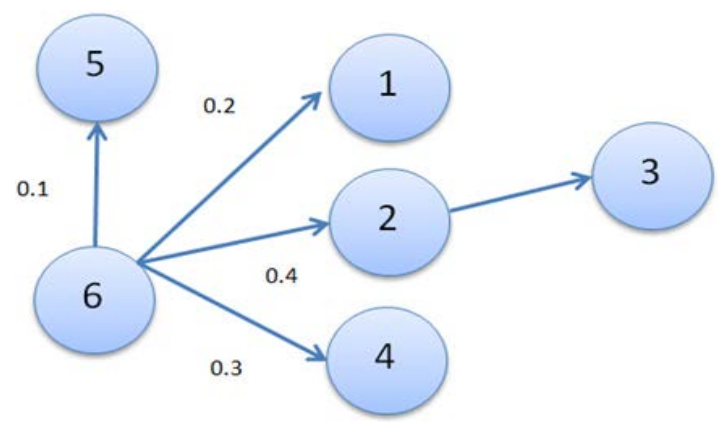

Figure 1. Schematic diagram of the page Jump. 
It is shown in Figure 3, the atmospheric science background students have demands for the resource, so the platform should provide more meteorological resources, in accordance with professional background to create resource classification system, based on user authentication information, combined with professional background, providing personalized service device.

\section{Conclusions}

The application of data mining technology can improve the resource organization mode, for the learners use it conveniently and quickly. Such as the standard of data mining language, on the effective mining method of multiple types of knowledge, and how to data mining in Internet network fast and effectively. With the deepening of the study, data mining have a greater impact on the data mining technology is bound to the resource library construction.

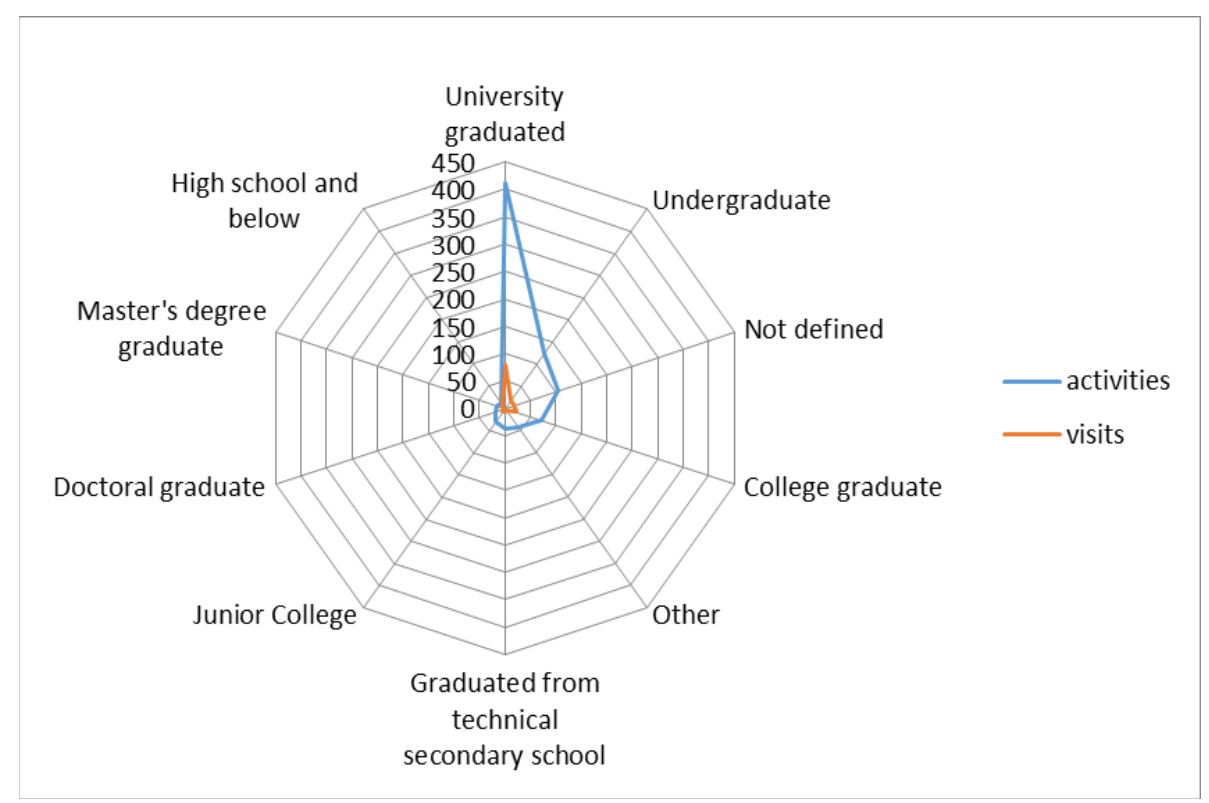

Figure 2. Education information radar chart.

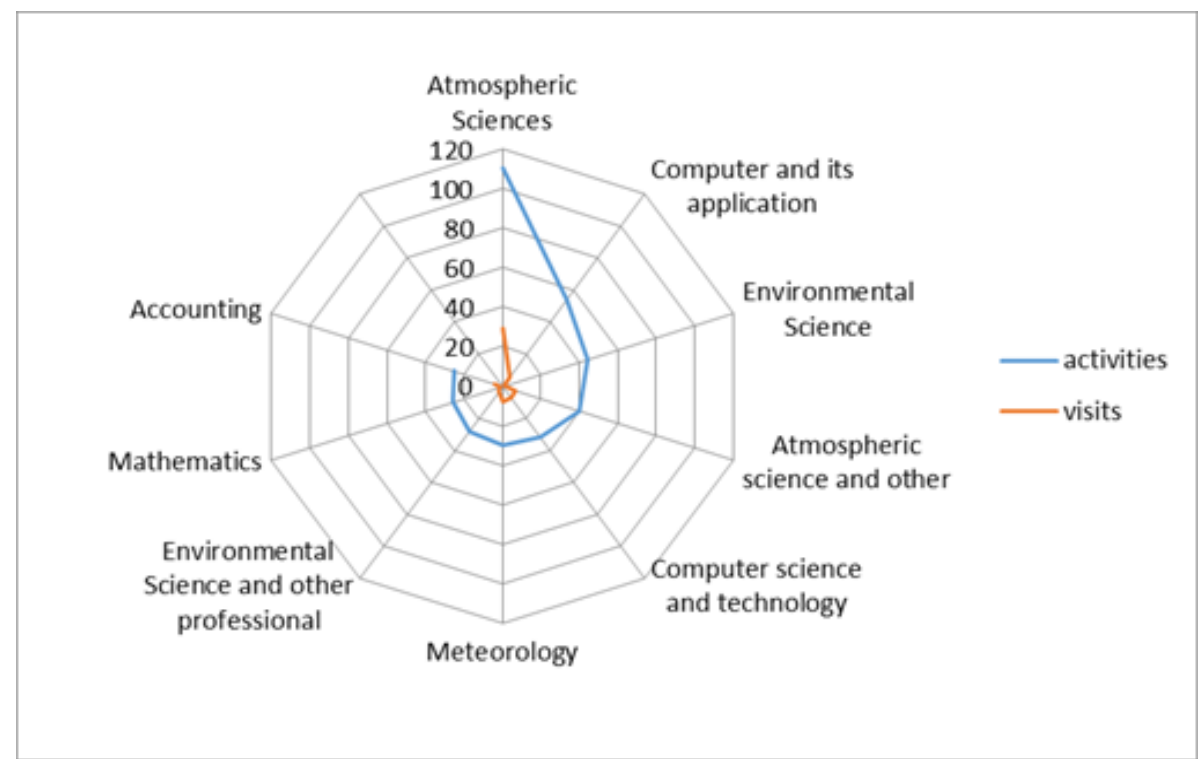

Figure 3. Professional information radar chart. 
In the practical application process, there are two important problems of educational resources: 1) The learning process is not suitable for individual learning is the fundamental problems of current network resources. 2) Resource retrieval difficulties, download time, resource library organization structure, the search query function setting is another cause of this phenomenon.

\section{References}

[1] Romero, C. and Ventura, S. (2007) Educational Data Mining: A Survey from 1995 to 2005. Expert Systems with Applications, 33, 125-146. http://dx.doi.org/10.1016/j.eswa.2006.04.005

[2] Romero, C., Ventura, S. and Garcia, E. (2005) Data Mining in Course Management Systems: Moodle Case Study and Tutorial. Computers \& Education, 51, 368-384. http://dx.doi.org/10.1016/j.compedu.2007.05.016

[3] Ge, D.K., Zhang, S.G. and Wei, S.P. (2012) The Educational Data Mining: Method and Application. Educational Science Publishing House, Beijing, 29-30.

[4] Zhang, X. (2012) Based on the Strategy of Cyber Source of Basic Education Effective Development and Innovation of Application of Data Mining in Beijing. Shaanxi Normal University.

[5] Baker, and Yacef. The State of Educational Data Mining in 2009: A Review and Future Visions [EB/OL].

[6] Siemens, G. Learning and Knowledge Analytics-Knewt on-the Future of Education? http://www.learning-analytics.net/?p=126.2011

[7] (2012) Big Data for Development: Challenges \& Opportunities. http://www.unglobalpulse.org/sites/default/files/BigDataforDevelopment-UNGlobalPulseJune2012.pdf 